\section{Sal 14}

INDICATIONS OF MANDIBLE BONE RESECTION IN CASES OF MALIGNANT TUMORS OF THE PAROTID GLAND R. SCHMELZLE and N. SCHWENZER

The indications of the mandible bone resection depend on the tumors location, the tumors diameter and the histological findings. After a review about our results of 15 years surgery of malignant tumors and metastases of the parotid gland, the neighbourhood of those tumors close to the mandible bone is demonstrated. Particularly the importance of tumors invading the temporo-mandibular joint is pointed out. The success of mandible bone resection depends first at all upon an early diagnoses in the field of the stomatognathic system. Our clinical findings show the superiority of modern diagnostic proceedings preoperatively and in the follow up period. The developements of bone reconstruction are a 1 so demonstrated, especia11y the modern microsurgical methods of iliac bone transplantation. The vessel anatomy in the maxillo-facial and groin regions and the blood supply of the iliac bone show, several variations. Nevertheless surgical preparation of the composite iliac flap for mandible bone reconstruction nowadays is one of the best methods. The bone transplantation has sometimes to be combined with simultaneous microsurgical reconstruction of the alveolaris inferior nerve. The possibility of tumorresection and one stage reconstruction of bone and soft tissues has to be discussed. Klinik für Kiefer- und Gesichtschirurgie der Universität Tübingen, Osianderstraße 2-8, D 7400 Tübingen

\section{Sal 15}

THE SIGNIFICANCE AND DIFFERENTIATION OF MALIGNANT SALIVARY GIAND TUMORS.A REVIEW OF 130 CASES. St.W.Bonorden and E.Machtens.

In a period of 13 years (1972-1985) 428 patients with pathology of the salivary glands were treated at the university hospital "Knappschafts-Krankenhaus" in BochumLangendreer.

With the exception of 55 patients with inflammatory pathology, the remaining 373 patients presented 243 cases (i.e. 65.14 ) benignant and 130 cases (i.e. 34.8\%) malignant lesions. The majority (109 or 29.22\%) of the malignant lesions were of epithelial origin as compared with lesions of non-epithelial origin (17 or 4.5\%) and salivary gland associated or metastatic tumors ( 4 or $1.07 \%$ ). The high incidence of malignancy in salivary gland tumors makes subtile pretherapeutic diagnostics mandatory; this may ensure during the first operative intervention the complete extirpation of the malignant tumor. If the latter is not possible feasible, the histomorphology of the malignancy should be identified and the size of the lesion reduced as much as possible. The best results were obtained in conjunction with additional radio- and chemotherapy. The significance of modern diagnostic procedures such as computerised tomography and magnetic resonance will be pointed out. This is especially important in cases of tumor recurrence or when tumors are localised in regions less accessible to routine diagnostic procedures.

In our department the therapeutic principle is, when possible, the primary radical surgery. In case of carcinoma this is enhanced by the suprahyoid and/or the radical neckdissection. This is subsequently followed by tumoricidal radiotherapy.

Abteilung für Mund-,Kiefer- und Gesichtschirurgie -plastische Operationen-,Knappschaftskrankenhaus -Universitätsklinik-, In der Schornau 23-25,D-4630 Bochum - 7.

\section{Sal 16}

ACINIC CELL TUMOR VERSUS ACINIC CELL CARCINOMA; OUR PRESENT KNOWLEDGE OF THIS TUMOP W. -j. Höltje, B. Henschel

The first reports on a salivary gland tumor with light colored cells were published by NASSE in 1892 who gave a histologic description of the acinic cell tumor and distinguished it from other salivary gland tumors.

Since the early fifties, about 100 reports on the clinical course of this rare salivary gland tumor have been published, covering approxemately 860 cases.

A number of important questions regarding its benign or malignant nature and its prognosis have not been solved up to now. Opinions regarding its therapy are also diverging considerably.

On the basis of four own cases and the reports on the clinical courses in the literature it is tried to sum up our knowledge of the acinic cell tumor / carcinoma and a therapeutical concept is proposed: The acinic cell tumor must be considered a carcinoma and therefore calls for radical resection. If resection is not radical enough, the incidence of recurrences is high. Chemotherapy and radiotherapy are without any great effect.

Nordwestdeutsche Kieferklinik, UniversitätsKrankenhaus Eppendorf, Martinistr. 52 , 2000 Hamburg 20

\section{VHN 01}

BIOSTAIISTICAI. ASPECTS OF ADJUVANT CHEMOTHERAPY TRIALS. H. Platz 1) and M. Hudec 2) (DÖSAK) 3)

For the performance of controlled clinical trials the following claims are derived:

1) Necessity of adequate sample sizes:

The biostatistical evaluation of adjuvant therapy is rather difficult because the prognostic improvement due to adjuvant therapy is usually considered small and therefore requires large sample sizes to detect. To avoid too long accrual periods large trials frequently imply the collaboration of several clinical centres.

2) The importance of prognostic factors in trial design. Variability in prognosis due to prognostic factors is usually greater than beneficial prognostic effects due to adjuvant therapy. Neglecting well-known prognostic factors in controlled clinical trials leads not only to imprecise treatment comparisons, but furthermore results in biased and erroneous conclusions.

3) Techniques for utilizing prognostic factors in clinica1 trials A rather simple technique is to restrict a trial to one prognostically homogeneous subgroup of patients. But frequently problems arize from insufficient patient accrual, even in multicentric trials.

The dynamic modelling randomization technique due to ZELEN 1975 and MIETTINEN 1976 groups together patients with similar prognoses but different characteristics of prognostic factors.

Adaptive stratification techniques 1 ike that of POCOCK and SIMON 1975 try to achieve an overal1 balance of prognostic factors in each treatment group.

1) Allgemeines öffentliches Krankenhaus der Stadt Linz, Krankenhausstraße 9, A-4020 Linz

2) Institut für Statistik und Informatik der Univ. Wien, Rathausstraße 19/4, A-1010 Wien

3) Deutsch-österreichisch-Schweizerischer Arbeitskreis für Tumoren im Kiefer- und Gesichtsbereich 